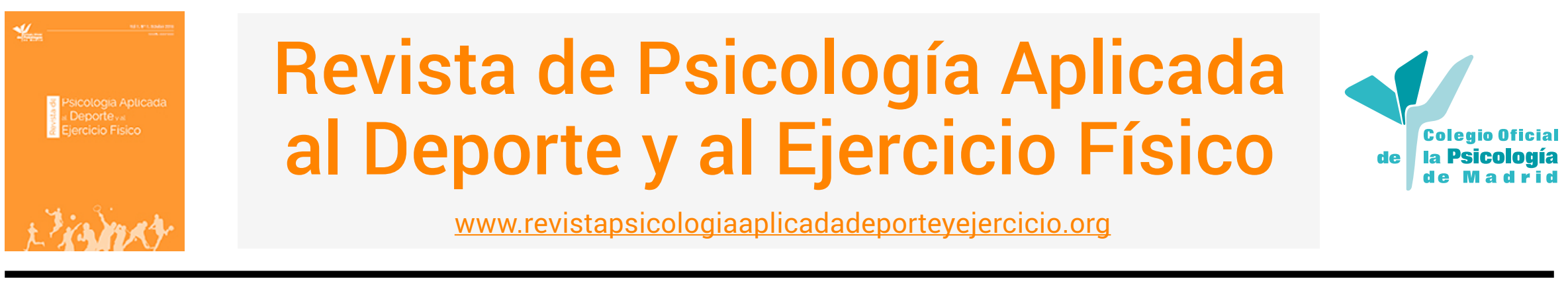

\title{
EDITORIAL
}

Nos complace compartir el último número del año 2021 de la Revista de Psicología Aplicada al Deporte y al Ejercicio Físico (RPADEF). Desde RPADEF, queremos dedicar este número a la memoria de los psicólogos y profesores Dr. Félix Guillén, Dr. José Carlos Caracuel y Dr. Jaume Cruz, que han contribuido al desarrollo y consolidación de la Psicología del Deporte en España, y lamentablemente, nos han dejado durante este año. Gracias por vuestro legado.

Además, desde RPADEF, queremos enviar un afectuoso saludo a todos los lectores y familiares que se han visto afectados por la COVID-19. Quisiéramos transmitiros muchos ánimos, fortaleza mental y la necesidad de extremar el cumplimiento de las medidas sanitarias para afrontar el futuro inmediato de forma segura, entre ellas, disfrutar de las fiestas navideñas con los seres queridos. Cuidémonos.

En relación a las actividades organizadas por la Sección de Psicología de la Actividad Física y el Deporte del Colegio Oficial de la Psicología de Madrid (COPM), tenemos que mencionar la III Jornada de Psicología del Deporte y Rendimiento Humano de la Comunidad de Madrid en la que participaron numerosos profesionales durante los días 19 y 20 de noviembre de 2021. En ella, hubo diferentes mesas redondas y conferencias sobre la Intervención Psicológica en esports, fútbol, baloncesto y otros deportes. Además, esta Jornada contó con la participación de la Asociación Española de Entrenadores de Baloncesto (AEEB), las escuelas de la Asociación de Futbolista Españoles (AFE), la Unión de Federaciones Deportivas Madrileñas (UFEDEMA) y la Asociación de Clubes de Esports (ACE). Podemos decir que fue todo un éxito. Gracias a todos/as los/as asistentes, colaboradores/ as y ponentes. Para ver una colección de fotos de la Jornada, se puede acceder al siguiente enlace: https://www. flickr. com/photos/copmadrid/albums/72157720179691767

En el transcurso de la clausura de la III Jornada, el colegio quiso rendir un merecido homenaje al psicólogo del deporte Pablo del Río por su trayectoria colegial y profesional. Pablo creo la unidad de Psicología en el Consejo Superior de
Deportes y lleva 30 años ejerciendo como coordinador del área, fue director de la Escuela Nacional de Entrenadores de Tenis, ha participado en 7 juegos Olímpicos, hace más de 1.300 sesiones al año con muchos de los deportistas que han conseguido grandes éxitos deportivos en los campeonatos del mundo, Juegos Olímpicos y otras competiciones nacionales e internacionales. Actualmente es el presidente de la Asociación Madrileña de Psicología del Deporte. El acto del homenaje se puede ver en el siguiente enlace de video:

\section{https://www. youtube.com/watch?v=w38XGhEh21g}

Otra actividad que ha tenido una excelente aceptación durante el año 2021, y que continuará durante el año 2022, es el II Ciclo de Conferencias Online en Psicología del Deporte, cuenta con un elevado número de asistentes en directo y abundantes visionados de los videos. Diferentes profesionales de la Psicología han compartido su trabajo dentro de este ciclo de conferencias. Las sesiones se programan los últimos lunes de cada mes a las 19.00 horas. Ver: $\quad$ https://www.copmadrid.org/web/formacion/actividades/20210723100127714438/ii-ciclo-videoconferencias-psicologia-deporte

Por otro lado, el 08 de febrero de 2022 se iniciará el XII Curso Online Experto en Psicología Coaching Deportivo. Su finalidad es que los/as psicólogos/as aprendan esta estrategia eficaz en modificación de la conducta, amplíen su repertorio de intervención y facilite su integración al mercado laboral en el deporte (ver: https://www.copmadrid. org/web/formacion/actividades/20210910165818164874/ sdo2202-experto-online-psicologia-coaching-deportivo).

También se iniciará el II Curso Online Experto en Psicología y Esports el 02 de marzo de 2022, con el propósito de especializar a los/as psicólogos/as en el sector y realizar intervenciones eficaces en esports (ver: https://www.copmadrid. org/web/formacion/actividades/20210805104727950510/ sdo2201-ii-edicion-experto-online-psicologia-esports).

En relación al presente número de RPADEF, nuestro objeto primordial es colaborar en la difusión en español de los tra- 
bajos de intervención, investigación aplicada, experiencias profesionales del psicólogo/a y revisiones sobre temas de actualidad con carácter aplicado en Psicología del Deporte, que se realizan en España y en el ámbito internacional. A continuación, se presentan los siguientes trabajos de autores/as con un excelente perfil profesional.

Dentro del apartado de Intervenciones en Psicología del Deporte, el primer artículo titulado "Formación en relajación para el control de estrés en boxeadores de un Centro de Tecnificación Deportiva", Aurelio Olmedilla, Isabel Moreno-Fernández, Belén Olmedilla-Caballero, Álvaro Sevilla y Verónica Gómez-Espejo plantean si las técnicas de relajación son herramientas útiles para controlar el estrés de jóvenes boxeadores y pueden extrapolarse a otros aspectos de su vida como al ámbito académico

El segundo artículo titulado "Desensibilización Sistemática para el control de la ansiedad: Un caso en atletismo", Rosendo Berengüí y María Á. Castejón proponen el objetivo de aplicar la técnica de desensibilización sistemática a una atleta de velocidad de 19 años que presentaba altos niveles de ansiedad competitiva.

El tercer artículo titulado "Intervención psicológica para mejorar el disfrute en una joven deportista de Fi Kid", Ana Sánchez Plazas y Enrique Cantón Chirivella pretenden evaluar la eficacia de una intervención psicológica para mejorar el rendimiento en una deportista de 13 años que practica Fit Kid.

Respecto al apartado de Investigaciones aplicadas en Psicología del Deporte, el cuarto artículo titulado "Rendimiento deportivo en atletas federados y su relación con autoestima, motivación e inteligencia emocional", Cristina Iglesias Suárez y María de la Villa Moral Jiménez analizan el rendimiento deportivo y su relación con la autoestima, la motivación y la inteligencia emocional en atletas federados.

El quinto artículo titulado "Espacios naturales, bienestar psicológico y satisfacción con la imagen corporal en corredores", Hannia Reyes-Rincón, Santiago Gibert-Isern, Sarai Ramírez-Colina, Eduardo Pineda-Arredondo, Vianey Guadalupe Argüelles-Nava y Yolanda Campos-Uscanga plantean el objetivo de determinar si existe relación entre correr en espacios naturales, bienestar psicológico, satisfacción con la imagen corporal y características de la actividad física.

En cuanto al apartado Revisión de temáticas de actualidad en Psicología del Deporte, el sexto artículo titulado "Relación entre resiliencia y rendimiento en deportistas. Revisión sistemática", Alicia María Sánchez Ortega y Francisco J. Ortín Montero establecen el objetivo revisar la evidencia empírica existente, sobre dicho constructo y su relación con el rendimiento de los deportistas, teniendo en cuenta diferentes factores, intrapersonales e interpersonales.
Además, contamos con una Entrevista de Antonio José de la Cuesta, psicólogo Experto en Psicología y Esports (COPM) e integrante del Grupo de Trabajo en Psicología, Videojuegos y Esports (COPM). Virginia Calvo es la entrevistada, COO y Co-Owner de Good Game Group, Secretaria Ejecutiva de la Asociación de Clubes de Esports y Co-CEO y Co-Owner en Giants Gaming Esports, una de las grandes impulsoras de los esports en España.

Y, por último, en el apartado de Recensión de libros, Diego Fuertes García, psicólogo del deporte de la Fundación Escuela de Mareo del Real Sporting de Gijón, realiza una revisión del libro "Un psicólogo en tu equipo: futbolistas de la cabeza a los pies" (año 2020, Madrid: Editorial Tutor), cuyo autor es el psicólogo del deporte Emilio González Nosti, director del área de Psicología del Real Sporting de Gijón.

Con el deseo de que disfruten durante la lectura de este nuevo número y aprendan de las experiencias aplicadas de los/as profesionales que han colaborado en el mismo, RPADEF les desea unas felices fiestas y que el año 2022 se mejor que el anterior, con salud, trabajo y mucha Psicología del Deporte.

Reciban un cordial saludo.
Alejo García-Naveira

Director de RPADEF 